could be the most important explanation of the observed heterogeneity in HIV epidemic in sub-Saharan Africa. Biomedical and some behavioural evidence have not sufficiently explained the variation. This study aimed at comparing the rate of concurrent sexual partnerships among adolescents in Arusha, Tanzania and Polokwane, South Africa, two areas with contrasting level of HIV magnitude. Methods A baseline cross-sectional study involving junior secondary school adolescents in Arusha, Tanzania and Polokwane South Africa was conducted in 2010 as part of the school-based Health Intervention project (LASH). Adolescents from 12 randomly selected schools from each country were interviewed regarding their sociodemographic characteristics, sexual behaviours and substance use. Data were entered and analysed using SPSS statistical package.

Results A total of 2408 adolescents from Arusha and 1649 from Polokwane participated in the study. Adolescent interviewed in Arusha were significantly older than their Polokwane counterpart (Mean age (SD) 19.3(0.5) and 15.5(0.7), respectively, $\mathrm{p}<0.001)$. The overall prevalence's of reported multiple concurrent sexual partnerships were $9.2 \%$ in Arusha and $25.2 \%$ in Polokwane (OR, 3.7, 95\% CI 3.0 to 4.7. Both males and Females adolescents from Polokwane were significantly more likely to report concurrent sexual partnerships than those from Arusha $(p<0.001)$. Moreover, adolescents from Polokwane reported practice of other HIV risk behaviours as compared to those in Arusha $(p<0.001)$. History of sexual experience (vaginal, oral, anal), ever use of condom and substance use were independent predictors of multiple concurrent sexual partnerships in both sites.

Conclusions High practice of multiple concurrent sexual partnerships among adolescent living in high HIV prevalent area as compared to those in low prevalence area indicate that concurrent partnership could be the most important explanation of the observed heterogenic HIV transmission in sub-Saharan Africa. Being sexually active and substance use predict practice of concurrent sexual partnerships among adolescents. Delaying sexual debut and addressing substance use among adolescents may have a significant impact on HIV epidemic.

\section{P1-S5.36 CONCURRENCY AMONG WOMEN <25 YEARS OF AGE SCREENED IN FAMILY PLANNING AND REPRODUCTIVE HEALTH CLINICS IN US PUBLIC HEALTH SERVICE REGION X, JANUARY 2009-JUNE 2010}

doi:10.1136/sextrans-2011-050108.214

W Nakatsukasa-Ono, D Fine. Center for Health Training, Seattle, USA

Background To better understand the dynamics of STIs and HIV, researchers are increasingly examining the determinants and consequences of sexual networks. In January 2009, the Infertility Prevention Project (IPP) in US Public Health Service Region X-one of 10 regional projects funded by the Centers for Disease Control and Prevention (CDC)-began collecting a new network sexual risk behaviour for IPP chlamydia (CT) tests. The Region X IPP decided to collect sex partner (SP) concurrency based on the literature, research conducted in California, CDC input and regional interest.

Methods CT positivity (CT+) was calculated by demographics, sexual risk behaviours, SP concurrency and clinical findings for 94,433 tests among women $<25$ years of age screened in Region X IPP family planning and reproductive health (FP/RH) clinics from January 2009 to June 2010. Univariate and multivariate analyses were conducted.

Results Of the 94433 tests, $62 \%$ were age 20-24, and $72 \%$ were non-Hispanic white. 14\% of clients reported their SPs definitely, $41 \%$ reported "possibly" and 38\% reported it was "unlikely" their SPs had concurrent SPs in the last 12 months. CT + ranged from $7.6 \%$ among clients reporting their SPs "definitely" had concurrent SPs to $5.2 \%$ among those reporting it was "unlikely." Clients reporting their SPs "definitely" or "possibly" had concurrent SPs were more likely to report other sexual risk behaviours and have clinical findings on examination. Significant $(p<0.001)$ factors for CT+ included SP concurrency (definitely: $A O R=1.20$; possibly: $\mathrm{AOR}=1.25)$; age (15-19 years: $\mathrm{AOR}=1.34)$; race/ethnicity (Black: $\mathrm{AOR}=1.55$; American Indian/Alaska Native: $\mathrm{OR}=1.60$; Native Hawaiian/Other Pacific Islander: $A O R=1.92)$; CT infection in the last 12 months $(\mathrm{AOR}=2.06)$; new SP $(\mathrm{AOR}=1.43)$, multiple SPs $(\mathrm{AOR}=1.48)$, symptomatic $\mathrm{SP}(\mathrm{AOR}=4.07)$ in past 60 days; clinical findings (cervicitis or PID diagnoses; $\mathrm{AOR}=2.22$ ).

Conclusions It is feasible to collect SP concurrency with female clients in the context of a clinic visit. SP concurrency was a significant predictor of CT + beyond other risk factors. Results for clients who reported their SPs "possibly" had concurrent SPs may reflect clients being less knowledgeable about their SPs' sexual behaviours. Findings confirm the importance of assessing network characteristics such as SP concurrency, the need to incorporate SP concurrency in risk reduction counselling, and the potential to use this measure in empirically based screening decisions.

\section{P1-S5.37 EVOLUTION OF SEXUAL NETWORKS OVER TIME IN MANITOBA, CANADA}

doi:10.1136/sextrans-2011-050108.215

A Jolly. Public Health Agency of Canada, Ottawa, Canada

Background Recent increases in bacterial STI in Canada and the resurgence of syphilis require further investigations into the transmission of STI. Specifically, adaptation of sexual networks and sexually transmitted pathogens to prevention efforts such as screening and partner notification have been proposed as a major factor in persistence of STI. We investigate the size and morphology of sexual networks generated by routinely collected contact tracing data for gonorrhoea, chlamydia and syphilis for the whole province of Manitoba, at three time points; 1990-1992; 1997-1998, and last, from 2002 to 2003.

Methods We compared the sizes of the components in which cases and contacts were connected by sexual intercourse at the three time points, and cross matched chart numbers of the cases from 1990-1992 with those from 1997-1998 in order to identify which individuals were active within specific networks $8-10$ years later. We reviewed the changes in the networks along with the rates over time in order to assess the epidemic phases of the pathogen and their possible effects on the networks.

Results From 1990 to 1992, 20223 cases were available for analysis; from a 6-month period in 1997-1998, 4544 cases and contacts were

A

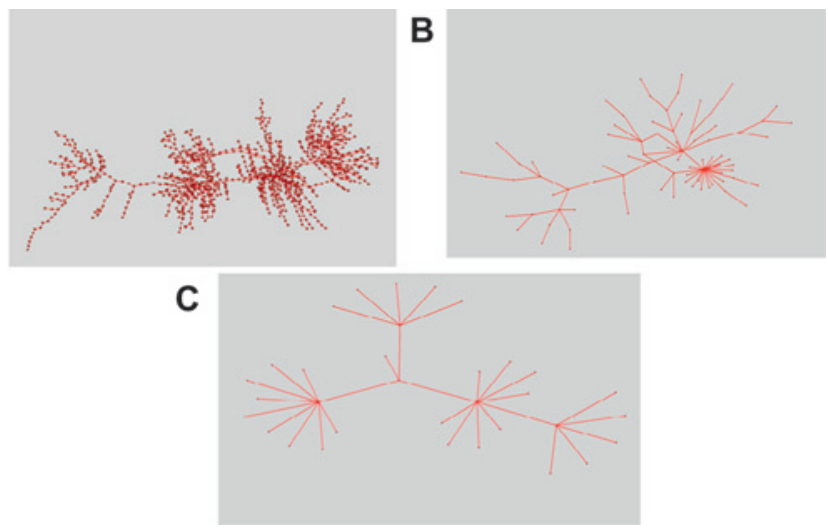

Abstract P1-S5.37 Figure 1 Evolution of sexual networks over time in Manitoba, Canada A) Pruned largest component, $n=2,166,1990-1992$, B) Largest component $n=82,1997-1998$ and C) largest component $\mathrm{n}=33$, 2003-2004. 